\title{
Vieta-Pell and Vieta-Pell-Lucas polynomials
}

\section{Dursun Tasci and Feyza Yalcin ${ }^{*}$}

${ }^{\text {"Correspondence: }}$

fyalcin@gazi.edu.tr

Department of Mathematics,

Faculty of Science, Gazi University,

Ankara, 06500, Turkey

\begin{abstract}
In the present paper, we introduce the recurrence relation of Vieta-Pell and Vieta-Pell-Lucas polynomials. We obtain the Binet form and generating functions of Vieta-Pell and Vieta-Pell-Lucas polynomials and define their associated sequences. Moreover, we present some differentiation rules and finite summation formulas.

MSC: Primary 11C08; secondary 11B39

Keywords: Vieta-Pell; Vieta-Pell-Lucas polynomials
\end{abstract}

\section{Introduction}

Andre-Jeannin [1] introduced a class of polynomials $U_{n}(p, q ; x)$ defined by

$$
U_{n}(p, q ; x)=(x+p) U_{n-1}(p, q ; x)-q U_{n-2}(p, q ; x), \quad n \geq 2,
$$

with the initial values $U_{0}(p, q ; x)=0$ and $U_{1}(p, q ; x)=1$.

Vieta-Lucas polynomials were studied as Vieta polynomials by Robbins [2]. VietaFibonacci and Vieta-Lucas polynomials are defined by

$$
\begin{aligned}
& V_{n}(x)=x V_{n-1}(x)-V_{n-2}(x), \quad n \geq 2, \\
& v_{n}(x)=x v_{n-1}(x)-v_{n-2}(x), \quad n \geq 2,
\end{aligned}
$$

respectively, where $V_{0}(x)=0, V_{1}(x)=1$ and $v_{0}(x)=2, v_{1}(x)=x$ [3]. The recursive properties of Vieta-Fibonacci and Vieta-Lucas polynomials were given by Horadam [3].

For $p=0$ and $q=1$, Vieta-Fibonacci polynomials are a special case of the polynomials $U_{n}(p, q ; x)$ in [1]. Further, $U_{n, m}(p, q ; x)$ in [4] for $p=0, q=1, m=2$ gives Vieta-Fibonacci polynomials.

Chebyshev polynomials are a sequence of orthogonal polynomials which can be defined recursively. Recall that the $n$th Chebyshev polynomials of the first kind and second kind are denoted by $T_{n}(x)$ and $U_{n}(x)$, respectively.

It is well known that the Chebyshev polynomials of the first kind and second kind are closely related to Vieta-Fibonacci and Vieta-Lucas polynomials. So, in [5] Vitula and Slota redefined Vieta polynomials as modified Chebyshev polynomials. The related features of Vieta and Chebyshev polynomials are given as

$$
\begin{aligned}
& V_{n}(x)=U_{n}\left(\frac{1}{2} x\right) \quad[3], \\
& v_{n}(x)=2 T_{n}\left(\frac{1}{2} x\right) \quad(\text { see }[2,6]) .
\end{aligned}
$$

(c) 2013 Tasci and Yalcin; licensee Springer. This is an Open Access article distributed under the terms of the Creative Commons Attribution License (http://creativecommons.org/licenses/by/2.0), which permits unrestricted use, distribution, and reproduction in any medium, provided the original work is properly cited. 
For $|x|>1$, we consider $t_{n}(x)$ and $s_{n}(x)$ polynomials by the following recurrence relations:

$$
\begin{array}{ll}
t_{n}(x)=2 x t_{n-1}(x)-t_{n-2}(x), & n \geq 2, \\
s_{n}(x)=2 x s_{n-1}(x)-s_{n-2}(x), & n \geq 2,
\end{array}
$$

where $t_{0}(x)=0, t_{1}(x)=1$ and $s_{0}(x)=2, s_{1}(x)=2 x$. We call $t_{n}(x)$ the $n$th Vieta-Pell polynomial and $s_{n}(x)$ the $n$th Vieta-Pell-Lucas polynomial.

The relations below are obvious

$$
\begin{aligned}
& s_{n}(x)=2 T_{n}(x), \\
& t_{n+1}(x)=U_{n}(x) .
\end{aligned}
$$

The first few terms of $t_{n}(x)$ and $s_{n}(x)$ are as follows:

$$
\begin{array}{ll}
t_{2}(x)=2 x, & s_{2}(x)=4 x^{2}-2, \\
t_{3}(x)=4 x^{2}-1, & s_{3}(x)=8 x^{3}-6 x, \\
t_{4}(x)=8 x^{3}-4 x, & s_{4}(x)=16 x^{4}-16 x^{2}+2, \\
t_{5}(x)=16 x^{4}-12 x^{2}+1, & s_{5}(x)=32 x^{5}-40 x^{3}+10 x, \\
t_{6}(x)=32 x^{5}-32 x^{3}+6 x, & s_{6}(x)=64 x^{6}-96 x^{4}+36 x^{2}-2, \\
t_{7}(x)=64 x^{6}-80 x^{4}+24 x^{2}-1, & s_{7}(x)=128 x^{7}-224 x^{5}+112 x^{3}-14 x .
\end{array}
$$

The aim of this paper is to determine the recursive key features of Vieta-Pell and VietaPell-Lucas polynomials. In conjunction with these properties, we examine their interrelations and define their associated sequences. Furthermore, we present some differentiation rules and summation formulas.

\section{Main results}

Some fundamental recursive properties of Vieta-Pell and Vieta Pell-Lucas polynomials are given in this section.

\section{Characteristic equation}

Vieta-Pell and Vieta-Pell-Lucas polynomials have the following characteristic equation:

$$
\lambda^{2}-2 x \lambda+1=0
$$

with the roots $\alpha$ and $\beta$

$$
\begin{aligned}
& \alpha=x+\sqrt{x^{2}-1}, \\
& \beta=x-\sqrt{x^{2}-1} .
\end{aligned}
$$

Also, $\alpha$ and $\beta$ satisfy the following equations:

$$
\begin{aligned}
& \alpha+\beta=2 x, \\
& \alpha \beta=1, \\
& \alpha-\beta=\Delta=2 \sqrt{x^{2}-1} .
\end{aligned}
$$




\section{Binet form}

By appropriate procedure, we can easily find the Binet forms as

$$
\begin{aligned}
& t_{n}(x)=\frac{\alpha^{n}-\beta^{n}}{\alpha-\beta}, \\
& s_{n}(x)=\alpha^{n}+\beta^{n} .
\end{aligned}
$$

\section{Generating function}

Vieta-Pell and Vieta-Pell-Lucas polynomials can be defined by the following generating functions:

$$
\begin{aligned}
& \sum_{n=0}^{\infty} t_{n}(x) y^{n}=y\left(1-2 x y+y^{2}\right)^{-1} \\
& \sum_{n=0}^{\infty} s_{n}(x) y^{n}=(2-2 x y)\left(1-2 x y+y^{2}\right)^{-1} .
\end{aligned}
$$

\section{Negative subscript}

We can also extend the definition of $t_{n}(x)$ and $s_{n}(x)$ to the negative index

$$
\begin{aligned}
& t_{-n}(x)=-t_{n}(x), \\
& s_{-n}(x)=s_{n}(x) .
\end{aligned}
$$

\section{Simson formulas}

$$
\begin{aligned}
& t_{n+1}(x) t_{n-1}(x)-t_{n}^{2}(x)=-1, \\
& s_{n+1}(x) s_{n-1}(x)-s_{n}^{2}(x)=4\left(x^{2}-1\right) .
\end{aligned}
$$

We arrange the first ten coefficients of $t_{n}(x)$ in Table 1 . Let $T(n, j)$ denote the element in row $n$ and column $j$, where $j \geq 0, n \geq 1$. As seen from the Table 1 , it is obvious that

$$
T(n, 0)=2 T(n-2,0)+T(n-1,0)
$$

Table 1 The first ten coefficients of $t_{n}(x)$

\begin{tabular}{lrrrrr}
\hline $\boldsymbol{n} \bigvee \boldsymbol{j}$ & $\mathbf{0}$ & $\mathbf{1}$ & $\mathbf{2}$ & $\mathbf{3}$ & $\mathbf{4}$ \\
\hline 0 & 0 & & & & \\
1 & 1 & & & & \\
2 & 2 & & & & \\
3 & 4 & -1 & & & \\
4 & 8 & -4 & & & \\
5 & 16 & -12 & 1 & & \\
6 & 32 & -32 & 6 & & \\
7 & 64 & -80 & 24 & -1 & \\
8 & 128 & -192 & 80 & -8 & \\
9 & 256 & -448 & 240 & -40 & 1 \\
\hline
\end{tabular}


can be written like the coefficients of Pell polynomials in [7]. Moreover,

$$
\sum_{j=0}^{\left\lfloor\frac{n-1}{2}\right\rfloor} T(n, j)=n
$$

For example, for $n=8$ we can find

$$
\sum_{j=0}^{3} T(8, j)=T(8,0)+T(8,1)+T(8,2)+T(8,3)=8 .
$$

Let $P_{n}$ denote the $n$th Pell number, so we have

$$
\sum_{j=1}^{\left\lfloor\frac{n-1}{2}\right\rfloor}|T(n, j)|=P_{n}
$$

\subsection{Interrelations of $t_{n}(x)$ and $s_{n}(x)$}

Most of the equations below can be obtained by using the Binet form and convenient routine operations

$$
\begin{aligned}
& t_{n+1}(x)-t_{n-1}(x)=s_{n}(x)=2 x t_{n}(x)-2 t_{n-1}(x), \\
& s_{n+1}(x)-s_{n-1}(x)=4\left(x^{2}-1\right) t_{n}(x), \\
& t_{n}(x) s_{n}(x)=t_{2 n}(x), \\
& s_{n}^{2}(x)+4\left(x^{2}-1\right) t_{n}^{2}(x)=2 s_{2 n}(x), \\
& s_{n}^{2}(x)-4\left(x^{2}-1\right) t_{n}^{2}(x)=4, \\
& t_{n+1}^{2}(x)-t_{n}^{2}(x)=t_{2 n+1}(x), \\
& s_{n+1}^{2}(x)-s_{n}^{2}(x)=4\left(x^{2}-1\right) t_{2 n+1}(x), \\
& s_{n+1}^{2}(x)+s_{n}^{2}(x)=2 x s_{2 n+1}(x)+4, \\
& t_{n+1}^{2}(x)-t_{n-1}^{2}(x)=2 x t_{2 n}(x), \\
& s_{n}(x) s_{n+1}(x)-4\left(x^{2}-1\right) t_{n}(x) t_{n+1}(x)=4 x, \\
& s_{n}(x) s_{n+1}(x)+4\left(x^{2}-1\right) t_{n}(x) t_{n+1}(x)=2 s_{2 n+1}(x), \\
& s_{4 n}(x)-2=4\left(x^{2}-1\right) t_{2 n}^{2}(x), \\
& t_{n+1}(x)-x t_{n}(x)=\frac{1}{2} s_{n}(x), \\
& 2 s_{n+1}(x)-2 x s_{n}(x)=4\left(x^{2}-1\right) t_{n}(x) .
\end{aligned}
$$

Proposition $1 s_{n}\left(2 x^{2}-1\right)-s_{n}^{2}(x)=-2$.

Proof Consider the expression $s_{n}\left(2 x^{2}-1\right)$. Then $\alpha, \beta, \Delta$ are replaced by $\alpha^{*}, \beta^{*}, \Delta^{*}$, respectively. So, $\alpha^{*}=\alpha^{2}, \beta^{*}=\beta^{2}, \Delta^{*}=2 x \Delta$ and by using the Binet form, the proof is completed. 


\subsection{Associated sequences}

Definition 1 The $k$ th associated sequences $\left\{t_{n}^{(k)}(x)\right\}$ and $\left\{s_{n}^{(k)}(x)\right\}$ of $\left\{t_{n}(x)\right\}$ and $\left\{s_{n}(x)\right\}$ are defined by, respectively $(k \geq 1)$

$$
\begin{aligned}
& t_{n}^{(k)}(x)=t_{n+1}^{(k-1)}(x)-t_{n-1}^{(k-1)}(x), \\
& s_{n}^{(k)}(x)=s_{n+1}^{(k-1)}(x)-s_{n-1}^{(k-1)}(x),
\end{aligned}
$$

where $t_{n}^{(0)}(x)=t_{n}(x)$ and $s_{n}^{(0)}(x)=s_{n}(x)$.

Presently,

$$
\begin{aligned}
& t_{n}^{(1)}(x)=s_{n}(x) \quad(\text { by }(4)), \\
& s_{n}^{(1)}(x)=\Delta^{2} t_{n}(x) \quad(\text { by }(5))
\end{aligned}
$$

are the members of the first associated sequences $\left\{t_{n}^{(1)}(x)\right\}$ and $\left\{s_{n}^{(1)}(x)\right\}$. If (6) and (7) are applied repeatedly, the results emerge

$$
\begin{aligned}
& t_{n}^{2 j}(x)=s_{n}^{(2 j-1)}(x)=\Delta^{2 j} t_{n}(x), \\
& t_{n}^{(2 j-1)}(x)=s_{n}^{(2 j-2)}(x)=\Delta^{2 j-2} s_{n}(x) .
\end{aligned}
$$

\section{Some special values of $t_{n}(x)$ and $s_{n}(x)$}

$$
\begin{aligned}
& \left\{\begin{array}{l}
t_{n}(-x)=(-1)^{n+1} t_{n}(x), \\
t_{n}(1)=n, \\
t_{n}(-1)=(-1)^{n+1} n, \\
t_{2 n}(0)=0, \\
t_{2 n-1}(0)=(-1)^{n+1},
\end{array}\right. \\
& \left\{\begin{array}{l}
s_{n}(1)=2, \\
s_{n}(-1)=2(-1)^{n}, \\
s_{2 n}(0)=2(-1)^{n}, \\
s_{2 n-1}(0)=0 .
\end{array}\right.
\end{aligned}
$$

\subsection{Differentiation formulas}

$$
\begin{aligned}
& \frac{d s_{n}(x)}{d x}=2 n t_{n}(x), \\
& \frac{d t_{n}(x)}{d x}=\frac{n s_{n}(x)-2 x t_{n}(x)}{2\left(x^{2}-1\right)}, \\
& \frac{d^{2} s_{n}(x)}{d x^{2}}=n\left(\frac{n s_{n}(x)-2 x t_{n}(x)}{x^{2}-1}\right) .
\end{aligned}
$$

Since the derivation function of $s_{n}(x)$ is a polynomial, all of the derivatives must exist for all real numbers. Thus, we can give the following formulas. 


\section{Proposition 2}

$$
\begin{aligned}
& \left.\frac{d^{2} s_{n}(x)}{d x^{2}}\right|_{x=1}=\frac{2}{3}\left(n^{4}-n^{2}\right), \\
& \left.\frac{d^{2} s_{n}(x)}{d x^{2}}\right|_{x=-1}=\frac{2}{3}(-1)^{n-1}\left(n^{2}-n^{4}\right) .
\end{aligned}
$$

Proof If we take the limit on $s_{n}^{\prime \prime}(x)=n\left(\frac{n s_{n}(x)-2 x t_{n}(x)}{x^{2}-1}\right)$, we have the numerical value of $s_{n}^{\prime \prime}$ at $x=1$ and $x=-1$.

$$
s_{n}^{\prime \prime}(1)=\frac{n}{2} \lim _{x \rightarrow 1} \frac{n s_{n}(x)-2 x t_{n}(x)}{(x-1)} .
$$

Since $s_{n}(1)=2, t_{n}(1)=n$, apply L'Hôpital's rule:

$$
\begin{aligned}
s_{n}^{\prime \prime}(1) & =\frac{n}{2} \lim _{x \rightarrow 1} \frac{\frac{d}{d x}\left(n s_{n}(x)-2 x t_{n}(x)\right)}{\frac{d}{d x}(x-1)} \\
& =\frac{n}{2} \lim _{x \rightarrow 1} \frac{d}{d x}\left(n s_{n}(x)-2 x t_{n}(x)\right) \\
& =\frac{n}{2} \lim _{x \rightarrow 1}\left(2 n^{2} t_{n}(x)-2 t_{n}(x)-2 x \frac{d}{d x} t_{n}(x)\right) \\
& =\frac{n}{2}\left(2 n^{2} t_{n}(1)-2 t_{n}(1)-2 \lim _{x \rightarrow 1} \frac{d}{d x} t_{n}(x)\right) \\
& =\frac{n}{2}\left(2 n^{3}-2 n\right)-\frac{1}{2} \lim _{x \rightarrow 1} \frac{d}{d x}\left(2 n t_{n}(x)\right) \\
& =n^{4}-n^{2}-\frac{1}{2} s_{n}^{\prime \prime}(1) .
\end{aligned}
$$

So, the proof for $x=-1$ is similar.

\subsection{Some summation formulas}

In this section we deal with the matrix

$$
\mathbf{V}=\left[\begin{array}{cc}
2 x & -1 \\
1 & 0
\end{array}\right]
$$

By induction, we have

$$
\mathbf{V}^{m}=\left[\begin{array}{cc}
t_{m+1}(x) & -t_{m}(x) \\
t_{m}(x) & -t_{m-1}(x)
\end{array}\right]
$$

So, the matrix V generates Vieta-Pell and Vieta-Pell-Lucas polynomials. Hence,

$$
\left[\begin{array}{c}
t_{m+1}(x) \\
t_{m}(x)
\end{array}\right]=\mathbf{V}^{m}\left[\begin{array}{l}
1 \\
0
\end{array}\right]
$$

and from (1.10) in [8], we get

$$
t_{m}(x)=\left[\begin{array}{ll}
1 & 0
\end{array}\right] \mathbf{V}^{m-1}\left[\begin{array}{l}
1 \\
0
\end{array}\right] .
$$


It is known that

$$
\mathbf{V}^{m+n}=\mathbf{V}^{m} \mathbf{V}^{n}
$$

From (8) and (11), the elementary formulas for $t_{n}(x)$ are obvious

$$
\begin{aligned}
& t_{m+n+1}(x)=t_{m+1}(x) t_{n+1}(x)-t_{m}(x) t_{n}(x), \\
& t_{m+n}(x)=t_{m+1}(x) t_{n}(x)-t_{m}(x) t_{n-1}(x), \\
& t_{m+n-1}(x)=t_{m}(x) t_{n}(x)-t_{m-1}(x) t_{n-1}(x) .
\end{aligned}
$$

If we use the matrix technique for summation in [8], we get the first finite summation as follows.

\section{Proposition 3}

(i) $\sum_{n=1}^{m} t_{n}(x)=\frac{t_{m+1}(x)-t_{m}(x)-1}{2(x-1)}$,

(ii) $\sum_{n=1}^{m} s_{n}(x)=\frac{s_{m+1}(x)-s_{m}(x)+2-2 x}{2(x-1)}$.

Proof (i) Let the matrix A,

$$
\mathbf{A}=\mathbf{I}+\mathbf{V}+\mathbf{V}^{2}+\cdots+\mathbf{V}^{n-2}+\mathbf{V}^{n-1}
$$

be the series of matrices. Then we have

$$
\mathbf{V A}=\mathbf{V}+\mathbf{V}^{2}+\cdots+\mathbf{V}^{n-1}+\mathbf{V}^{n}
$$

Hence,

$$
\begin{aligned}
& (\mathbf{V}-\mathbf{I}) \mathbf{A}=\mathbf{V}^{n}-\mathbf{I}, \\
& \mathbf{A}=(\mathbf{V}-\mathbf{I})^{-1}\left(\mathbf{V}^{n}-\mathbf{I}\right) \\
& =\frac{1}{2(x-1)}\left[\begin{array}{cc}
t_{n+1}(x)-t_{n}(x)-1 & t_{n-1}(x)-t_{n}(x)+1 \\
t_{n}(x)-t_{n-1}(x)-1 & t_{n-2}(x)-t_{n-1}(x)+2 x-1
\end{array}\right], \\
& \sum_{n=1}^{m} t_{n}(x)=\left[\begin{array}{ll}
1 & 0
\end{array}\right] \mathbf{A}\left[\begin{array}{l}
1 \\
0
\end{array}\right] \quad(\text { by }(10)) \\
& =\frac{1}{2(x-1)}\left[t_{m+1}(x)-t_{m}(x)-1 \quad t_{m-1}(x)-t_{m}(x)+1\right]\left[\begin{array}{l}
1 \\
0
\end{array}\right] \\
& =\frac{1}{2(x-1)}\left[t_{m+1}(x)-t_{m}(x)-1\right] \text {. }
\end{aligned}
$$

Thus, the proof is completed.

(ii)

$$
\begin{aligned}
\sum_{n=1}^{m} s_{n}(x) & =\sum_{n=1}^{m}\left(\alpha^{n}+\beta^{n}\right) \quad(\text { by }(3)) \\
& =\alpha\left(\frac{1-\alpha^{m}}{1-\alpha}\right)+\beta\left(\frac{1-\beta^{m}}{1-\beta}\right)
\end{aligned}
$$




$$
\begin{aligned}
& =\frac{(\alpha+\beta)-2 \alpha \beta-\left(\alpha^{m+1}+\beta^{m+1}\right)+\alpha \beta\left(\alpha^{m}+\beta^{m}\right)}{2-2 x} \\
& =\frac{2 x-2-\left(\alpha^{m+1}+\beta^{m+1}\right)+\alpha^{m}+\beta^{m}}{2(1-x)} \quad(\text { by }(1)) \\
& =\frac{s_{m+1}(x)-s_{m}(x)+2-2 x}{2(x-1)} \quad(\text { by }(3)) .
\end{aligned}
$$

This completes the proof.

Theorem 1 Let $\mathbf{V}$ be a square matrix such that $\mathbf{V}^{2}=2 x \mathbf{V}-\mathbf{I}$. Then, for all $n \in \mathbb{Z}^{+}$,

$$
\mathbf{V}^{n}=t_{n}(x) \mathbf{V}-t_{n-1}(x) \mathbf{I},
$$

where $t_{n}(x)$ is the $n$th Vieta-Pell polynomial and $\mathbf{I}$ is a unit matrix.

Proof The proof is obvious from induction.

\section{Competing interests}

The authors declare that they have no competing interests.

\section{Authors' contributions}

The authors declare that the research was realized in collaboration with the same responsibility and contributions. Both authors read and approved the final manuscript.

\section{Acknowledgements}

The authors thank the referees for their valuable suggestions, which improved the standard of the paper.

Received: 4 April 2013 Accepted: 11 July 2013 Published: 24 July 2013

\section{References}

1. Andre-Jeannin, R: A note on general class of polynomials. Fibonacci Q. 32(5), 445-454 (1994)

2. Robbins, N: Vieta's triangular array and a related family of polynomials. Int. J. Math. Math. Sci. 14, $239-244$ (1991)

3. Horadam, AF: Vieta polynomials. Fibonacci Q. 40(3), 223-232 (2002)

4. Djordjevic, GB: Some properties of a class of polynomials. Mat. Vesn. 49, 265-271 (1997)

5. Vitula, R, Slota, D: On modified Chebyshev polynomials. J. Math. Anal. Appl. 324, 321-343 (2006)

6. Jacobsthal, E: Über vertauschbare polynome. Math. Z. 63, 244-276 (1955)

7. Halici, S: On the Pell polynomials. Appl. Math. Sci. 5(37), 1833-1838 (2011)

8. Mahon, JM, Horadam, AF: Matrix and other summation techniques for Pell polynomials. Fibonacci Q. 24(4), 290-309 (1986)

\section{Submit your manuscript to a SpringerOpen ${ }^{\ominus}$ journal and benefit from:}

- Convenient online submission

Rigorous peer review

- Immediate publication on acceptance

Open access: articles freely available online

- High visibility within the field

- Retaining the copyright to your article 